\title{
Implications of CRISPR/Cas9 system in Hypertension and its related diseases
}

\author{
Durairaj Sekar $\mathbb{D}^{1} \cdot$ Ganesh Lakshmanan ${ }^{2} \cdot$ Biruntha $M^{3}$
}

Received: 31 March 2020 / Revised: 15 June 2020 / Accepted: 30 June 2020 / Published online: 9 July 2020

(c) Springer Nature Limited 2020

\section{To the Editor:}

CRISPR/Cas9 (Clustered Regularly Interspaced Short Palindromic Repeats and nuclease(s) associated to the CRISPR locus) is a genome editing system where guide RNA (gRNA) directs the Cas9 nuclease to create a doublestrand breaks (DSBs) in a specific place where it can be able to edit the specific portion of the genome using programmable nucleases with error prone or error free repair mechanisms [1, 2]. Many researchers argue genome editing is an authoritative and validated tool for medical research and that it provides new avenues for the treatment of hypertension and cardiovascular diseases [1-5].

Dysregulation of multiple genes added with environmental factors may cause non-communicable diseases like hypertension and cardiovascular diseases. In addition, mutations in the renin- angiotensin-aldosterone system (RAAS), impaired platelet function and thrombogenesis also induces hypertension and associated disease progressions. Moreover, cardiovascular risks are also attributed to hypertension progressions [3]. Researchers have done extensive research on understanding pathogenesis of hypertension and cardiovascular diseases, yet a curative treatment option is still elusive to control or manage the above said diseases.

Durairaj Sekar

duraimku@gmail.com

1 Dental Research Cell and Biomedical Research Unit (DRCBRULAC), Saveetha Dental College and Hospital, Saveetha Institute of Medical and Technical Science (SIMATS), Saveetha University, Chennai 600077, India

2 Department of Anatomy, Saveetha Dental College and Hospital, Saveetha Institute of Medical and Technical Science (SIMATS), Saveetha University, Chennai 600077, India

3 Department of Animal Health and Management, Alagappa University, Tamil Nadu 630003, India
Interestingly, across species and tissues, including cultured cells; CRISPR/Cas9 system allows manipulation of any gene at its own locus. It has been known that genetic and epigenetic alterations happen in the incongruous target region of the cellular system but still, it can be easily reverted by CRISPR/Cas9 platforms. Moreover, extensive efforts have been carried out to improve efficacy as well as target specificity of CRISPR/Cas9 systems. Epigenetic modification of certain genes in the cellular system achieved successfully through CRISPR/Cas9 systems [4]. Figure 1 Represents the CRISPR/CAS9 system editing the genome sequences in the cells. It is a realistic and simple technique to fuse with most of the genes such as acetyltransferase, lysine specific demethylase 1, Kruppel-associated box, DNA methyltransferases and ten-eleven translocation (TET) dioxygenase 1 and thus a potential agent for epigenomic modifications [4].

A recent study by Liu et al. [5] established a mouse model for Ace 2 deficiency in the treatment of hypertension with CRISPR/Cas9 technique, where, Ace2 gene knockout has been achieved by a vector with primers of single-guide RNA (gRNA) and then the transcribed gRNA/Cas9 mRNA is injected into the mouse zygote [5] suggesting that CRISPR/Cas9 system can be used to create a mouse model for the treatment of hypertension and associated diseases.

It has also been shown that using CRISPR/Cas9, researchers made genetically salt-sensitive hypertensive rat model to achieve genomic excision of G-protein coupled estrogen receptor (Gper1) gene for the treatment of hypertension and cardiovascular diseases. In addition, vascular relaxation is also noticed in the same rat model suggesting that Gperl is a candidate gene associated with increased vascular resistance thereby increasing the risk of inducing hypertension and cardiovascular diseases [6]. Interestingly, in an another study, they delivered a single short guide RNA using cardiac CRISPR/Cas9 based adeno-associated virus in the postnatal mice for the cardiovascular disease treatment [7]. The above studies clearly indicated that CRISPR/Cas9 based genome editing as an effective 


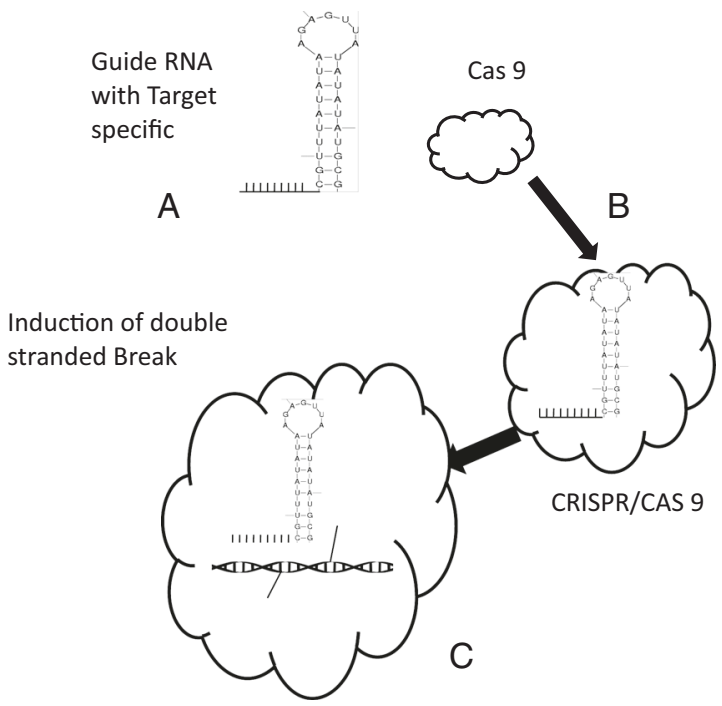

Fig. 1 Represents the CRISPR/CAS9 system editing the genome sequences in the cells. a Guide RNA is generated in the cells for specific site in the genome. b Cas9 prtein interact with Guide RNA specific sites. c CRISPR/Cas 9 complex introduce double stranded break in the target site.

modality in the treatment of hypertension and cardiovascular diseases.

In yet another observation, CRISPR/Cas9 system creates a mutation in microRNAs (miRNAs) binding site on its target gene to induce pro-inflammatory effects in the control of disease progressions. Such modifications on the target genes prevent the binding of miRNAs leading to down regulation of those genes ultimately reducing the disease progression itself [8]. CRISPR/Cas9 genome system is also used to create stroke-prone spontaneously hypertensive rats by constructing $\operatorname{Prdx} 2$ knockout (a gene coding an antioxidative enzyme), suggesting that $\operatorname{Prd} 22$ depletion causes a shorter life span with increased blood pressure in spontaneously hypertensive rats through augmented oxidative stress [9]. From the above points it is clearly understood that stable expression of genes in the cell systems encircling the hypertensive disorder as well as manipulation of those genes can be achieved by CRISPR/Cas9 system.

Cardiopulmonary disorders like pulmonary arterial hypertension (PAH) is also a commonly seen lethal condition and a recent research created a knockout model using CRISPR/Cas9 to prove that the loss of functional mutation on the gene potassium channel subfamily $\mathrm{K}$ member 3 $(K C N K 3)$ is responsible for PAH. This model will be useful in understanding the mechanism of PAH progression and also testing therapeutic molecules against PAH [10].

In the current scenario, novel treatment options are much more needed than ever for the management of life threatening diseases and to overcome the burden of debilitating diseases. An in-depth analysis of CRISPR/Cas9 systems may serve as a radical treatment option for many diseases such as cancer, autoimmune diseases, neurological disorders, hypertension and cardiovascular diseases. Many researchers have tried to establish the molecular mechanisms behind CRISPR/Cas9 system genome editing, but more results and clinical data are needed to demonstrate that the CRISPR/Cas9 editing system is a valid and reliable mode of treatment of the above said diseases and help to define its role in different medical treatments. In the treatment of hypertension and cardiovascular diseases using CRISPR/Cas9 system, the molecular basis behind it still remains elusive, mainly because the number of studies is sparse. More investigations with larger clinical samples will be needed to prove the above concepts in the treatment of hypertension and its related diseases.

Acknowledgements Sekar D is a recipient of the Extramural Grant (2019-0106/CMB/ADHOC/BMS), Indian Council of Medical Research (ICMR), Government of India, and their support is duly acknowledged. The authors also thankful to RUSA scheme Phase 2.0 grant [F-24-51/2014-U, Policy (TN Multi-Gen), Dept. of Edn, Govt. of India. Dt. 09.10.2018] for their financial support.

\section{Compliance with ethical standards}

Conflict of interest The authors declare that they have no conflict of interest.

Publisher's note Springer Nature remains neutral with regard to jurisdictional claims in published maps and institutional affiliations.

\section{References}

1. Rodríguez-Rodríguez DR, Ramírez-Solís R, Garza-Elizondo MA, Garza-Rodríguez ML, Barrera-Saldaña HA. Genome editing: a perspective on the application of CRISPR/Cas9 to study human diseases (Review). Int J Mol Med. 2019;43:1559-74. https://doi. org/10.3892/ijmm.2019.4112

2. Wright AV, Nuñez JK, Doudna JA. Biology and applications of CRISPR systems: harnessing nature's toolbox for genome engineering. Cell. 2016;164:29-44. https://doi.org/10.1016/j.cell. 2015.12.035

3. Sekar D. Circular RNA: a new biomarker for different types of hypertension. Hypertens Res. 2019;42:1824-5. https://doi.org/10. 1038/s41440-019-0302-y

4. Xie N, Zhou Y, Sun Q, Tang B Novel Epigenetic Techniques Provided by the CRISPR/Cas9 System. Stem Cells Int. 2018;2018:7834175. Published 2018 Jul. 10.1155/2018/7834175.

5. Liu C, Chen CY, Shang QH, Liu J. [Establishment of Ace2 knockout mouse model with CRISPR/Cas9 gene targeting technology]. Sheng Li XueBao. 2019;71:588-96. Chinese. PMID: 31440756

6. Waghulde H, Cheng X, Galla S, Mell B, Cai J, Pruett-Miller $\mathrm{SM}$, et al. Attenuation of microbiotaldysbiosis and hypertension in a CRISPR/Cas9 gene ablation rat model of GPER1. 
Hypertension. 2018;72:1125-32. https://doi.org/10.1161/ HYPERTENSIONAHA.118.11175

7. Johansen AK, Molenaar B, Versteeg D, Leitoguinho AR, Demkes C, Spanjaard B, et al. Postnatal cardiac gene editing using CRISPR/Cas9 With AAV9-mediated delivery of short guide RNAs results in mosaic gene disruption. Circ Res. 2017;121:1168-81. https://doi.org/10.1161/CIRCRESAHA.116. 310370

8. Jing W, Zhang X, Sun W, Hou X, Yao Z, Zhu Y. CRISPR/CAS9mediated genome editing of miRNA- 155 inhibits proinflammatory cytokine production by RAW264.7 cells. Biomed Res Int. 2015;2015:326042 https://doi.org/10.1155/2015/326042

9. Mahal Z, Fujikawa K, Matsuo H, Zahid HM, Koike M, Misumi $M$, et al. Effects of the Prdx2 depletion on blood pressure and life span in spontaneously hypertensive rats. Hypertens Res. 2019;42:610-7. https://doi.org/10.1038/s41440-019-0207-9

10. Lambert M, Capuano V, Boet A, Tesson L, Bertero T, Nakhleh MK, et al. Characterization of Kcnk3-mutated rat, a novel model of pulmonary hypertension. Circ Res. 2019;125:678-95. https:// doi.org/10.1161/CIRCRESAHA.119.314793 